Upcoming: August 1-6 Gordon Research Conf. on High-Temperature Materials, Processes and Diagnostics, Endorsed.

To list an event in the Calendar, contact J. Meiksin, Materials Research Society, 506 Keystone Drive, Warrendale, PA 150867573; 724-779-3004 ext. 522; fax 724-779-8313;

bulletin@mrs.org.

MRS, A-MRS, B-MRS, C-MRS, E-MRS, MRS-A, MRS-I, MRSJ, MRS-K, MRS-R, MRS-S, MRS-T, M-MRS, or IUMRS at the end of an entry indicates sponsorship or co-sponsorship of an event by the International Union of Materials Research Societies or one of its adhering bodies. "Endorsed" identifies events endorsed by MRS.

$\nabla$ identifies a new or revised entry this month.
See the May 2004 MRS BULLETIN for July 2004 Calendar entries. For updates, access www.mrs.org/ meetings/other/.

\section{AUGUST 2004}

1-6 Gordon Research Conf. on High-Temperature Materials, Processes and Diagnostics, Waterville, ME. Klaus Hilpert, Research Center Jülich, Jülich, Germany, 49-2461-61-3280; fax 492461-61-3699; e-mail k.hilpert@ fz-juelich.de. Endorsed.

2-4 $\nabla$ Intl. Surface Engineering Congress and Exhibition, Orlando, $F L$. ASM Customer Service, 9639 676-3290; fax 360-647-1445; e-mail 44073-0002; 800-336-5152

x5900; fax 440-338-4634; e-mail

Cust-srv@asminternational.org;

www.asminternational.org/surface. Physics, Tallahassee, FL. Y.-J. Wang,
2-5 11th Int Conf on High Magnetic Fields in Semiconductor, Berkeley, CA. P. Yu, University of Calfornia, Berkeley, CA, 94720; 510-642-8087; fax 510-643-8497; e-mail pyyu@|bl.gov.

2-6 16th Int. Conf. on High Magnetic Fields in Semiconductor 850-644-1496; fax 850-644-0534; e-mail wang@magnet.fsu.edu; www.semimag16.magnet.fsu.edu.

2-6 49th Annual SPIE Meeting, and Intl. Symp. on Optical Science and Technology, Denver, CO. SPIE, SPIE Meetings, P.O. Box 10

Bellingham, WA, 98227-0010; 360 spie@spie.org; spie.org/info/am. Growth (ICCG-14)/12th Intl. Conf. on Vapor Growth and Epitaxy

(ICVGE-12), Grenoble, France. Fabien Briola, 33-491-462556; e-mail iccg14exhibitors@ atout-org.com; iccg14.inpg.fr.
15-20 $\nabla$ L10 Ordered Intermetallic and Related Phases for Permanent Magnet and Recording Applications, Copper Mountain, CO. Michael McHenry, Data Storage Systems Center, Carnegie Mellon University, Pittsburgh, PA, 15213; 412-2682703; fax 412-268-3113; e-mailmm7g@andrew.cmu.edu; www.engconfintl.org/4ar.html.

22-25 COM2004: The Conf. of Metallurgists, Hamilton, ON. Ken Coley, McMaster Steel Research Centre, Hamilton, ON, L8S 4L7; 905-525-9140; fax 905526-8404; e-mail coleyk@mcmaster. ca; www.metsoc.org.

22-26 XIII Intl. Materials Research Congress, Cancun, Mexico. Col. Ladrillera de Benitez, 52-222211-4393; fax 52-222-211-4394; www.viep.buap.mx/imrc2004.htm. 
22-28 Intl. Symp. on Biological Polyesters (ISBP 2004), Beijing, Peoples Republic of China. Ya-Wu Wang, Tsinghua University, Beijing 100084, Peoples Republic of China, 86-10-62772676; fax 8610-62788784; e-mail microbes@ mail.tsinghua.edu.cn; microbes. biosci.tsinghua.edu.cn/ISBP.

23-25 4th Conf. on Synchrotron Radiation in Materials Science, Grenoble, France. Fabienne Mengoni, e-mail srms-4@esrf.fr; www.esrf.fr/Conferences/SRMS-4

23-273rd Intl. Conf on HWCVD (Cat-CVD) Process, Utrecht, Netherlands. Ruud Schropp, Utrecht University, Debye Inst, Princetonplein 5 Utrecht, The Netherlands; 31-30253-3170; fax 31-30-254-3165; e-mail R.E.I.Schropp@phys.uu.nl; www.hwcvd.nl.

23-27 9th Int. Conf. on Ferrites San Francisco, CA. Alex Goldman, Ferrite Technology Worldwide, 4625 Fifth Ave. \#305, Pittsburgh, PA 15213; e-mail ferritetec@aol.com; www.ceramics.org/meetings/ ferrites/ default.asp.

23-27 2004 IEEE Intl. Ultrasonics, Ferroelectrics, and Frequency Control 50th Anniversary Joint Conf., Montreal, Canada. R. Michael Garvey, Symmetricom, 34 Tozer Road, Beverly, MA; 01915 5510; 978-232-1417; fax 978-9274099; e-mail rmgarvey@ symmetricom.com; www.ieee-uffc.org

25-27 ASM Materials \& Processes for Medical Devices Conf. St. Paul, MN. ASM International, Materials Park, OH, 44073-0002; 440-338-5151; fax 440-338-4634; www.asminternational.org/ meddevices

25-27 UWEB 8th Summer Symp.-Bioengineered Blood Vessels 2004: Grafts, Stents, and Tissue Engineering, Seattle, WA Buddy Ratner, Univ. of Washington (UWEB), Seattle, WA, 98195-1720; e-mail info@uweb.engr.washington. edu; www uweb engr.washington. edu/news/sommersymposium.html.

28-3 World Renewable Energy Congress VIII (WREC VIII), Denver, CO. Ali Sayigh, PO Box 362, Brighton BN2 1YH, United Kingdom, 44-1273-625-643; fax 44-1273-625-768; e-mail asayigh@netcomuk.co.uk; www.wrenuk.co.uk.

\section{SEPTEMBER 2004}

1-2 RP Asia 2004-Promoting the Growth of Composites in Asia, Bangkok, Thailand. Nick Williams, 44-1743-241-289; fax 44-870 138-9539; www.rpasia.com.
4-9 $\boldsymbol{\nabla}$ 21st Intl. Conf. on Amorphous and Nanocrystalline Semiconductors, Lisbon, Portugal ICANS, ICANS 21 Campus da Caparica, 2829-516 Caparica, Portugal, 351-212-948-524/62; fax 351-212-941-365

e-mail icans21@fct.unl.pt; www.icans21.org.

5-10 Intl. Conf. on Polycrystalline Semiconductors-POLYSE 2004, Potsdam, Germany. Norbert Nickel, Hahn-Meitner-Institut Berlin, Kekulestr. 5, Berlin, Germany; 4930-67053-301; fax 49-30-67053333; e-mail nickel@hmi.de.

5-10 Joint European Magnetic Symposia - JEMS 2004, Dresden, Germany. Anke Kirchner, IFW Dresden, P.0. Box 270016 , Dresden, Germany, 49-351-4659405; fax 45-351-465-9541; e-mail jems04@ifw-dresden.de; www.ifw-dresden.de/imw/jems04.

6-9 Junior Euromat 2004, Lausanne, Switzerland. Anja Mangold, Hamburger Allee 26 , D-60486, Frankfurt, Germany; 49 69-7917-747; fax 49-69-7917-733 e-mail junior-euromat@fems.org; www.junior-euromat.fems.org.

6-10 E-MRS Fall 2004 Meeting, Warsaw, Poland. E-MRS Fall 04, email fall2004@e-mrs.org; www.emrs.org/meetings/fall2004. E-MRS

12-16 5th Intl. Conf. on High Temperature Ceramic Matrix Composites, Seattle, WA. The American Ceramic Society, P.0. Box 6136, Westerville, $\mathrm{OH}$ 43086-6136; 614-890-4700; fax 614-899-6109; e-mail info@ acers.org; www.acers.org.

12-17 $\nabla$ 15th European Conf. on Diamond, Diamond-Like Materials, Carbon Nanotubes, Nitrides \& Silicon Carbide, Riva Del Garda, Italy. April Williams, Elsevier, The Boulevard, Langford Lane, Kidlington, Oxford OX5 1GB, UK; 44-0-1865843089; fax 44-0-1865 843958; e-mail a.williams@elsevier.com; www.diamond-conference. elsevier.com.

13-17 Nano \& Giga Challenges in Microelectronics (NGCM 2004) Cracow, Poland. Anatoli Korkin, 480-539-4754; e-mail korkin@ nanoandgiga.com; www.asdn. net/ngcm2004. Endorsed.

13-17 Ninth Int. Conf. on Plasma Surface Engineering, Garmisch-Partenkirchen, Germany. Karin Reichel, VDI-Technology Center, Graf-Recke-Str. 84, D-40239, Duesseldorf, Germany; 49-211-6214-640; fax 49211-6214575-484; e-mail akplasma@vdi.de; www.akplasma.org/pse2004.php3.
15-17 $\nabla 2004$ Intl. Conf. on Solid State Devices and Materials, Tokyo, Japan. c/o Inter Group Corp., Conf. Secretariat, 4-9-17, Akasaka, Minato-ku, Tokyo, Japan, 81-3-3479-5131; fax 81-3-34231601; e-mail ssdm@intergroup.co.jp; www.ssdm.jp.

19-21 Fourth Intl. Conf. on Inorganic Materials, Antwerp, Belgium. Phillipa Fletcher, Bregor Winter Lane, West Hanney, Nr Wantage, Oxon, United Kingdom; 44-1235-868811; fax 44-1235868811; e-mail phillipa.fletcher@ dial.pipex.com; www. im-conference.com/contact.htm.

19-23 2nd Intl. Conf. on Environment-Induced Cracking of Metals (EICM-2), Banff, Alberta,

Canada. Sergei Shipilov, Banff, AB 403-220-4149; fax 403-282-8406; e-mail shipilov@enme.ucalgary.ca; www.enme.ucalgary.ca/EICM-2.

19-23 10th Intl. Symp. on Superalloys, Champion, PA. TMS Meeting Services, 184 Thorn Hill Rd., Warrendale, PA 15086; 724776-9000; fax 724-776-3770; e-mail mtgserv@tms.org; www.tms.org/ Meetings/Specialty/Superalloys2004/ SuperalloysHome.html.

20-21 Potentials and Risks of Nanoscale Materials, Augsburg, Germany. Bernd Stritzker, Universität Augsburg, Lehrstuhl Experimentalphysik 4, Institut fur Physik, D-86135 Augsburg, Germany; 49-821-5983400; fax 49-821-598-3425; e-mail stritzker@physik.uni-augsburg.de. Endorsed.

20-22 Boulder Damage Symp. XXXVI, Boulder, CO. SPIE, SPIE Meetings, P.0. Box 10, Bellingham, WA, 98227-0010; 360-676-3290; tax 360-647-1445 e-mail spie@spie.org; www.spie.org/events/bd.

20-25 13th Semi-Conducting and Insulating Materials Conference (SIMC-XIII-2004), Beijing, Peoples Republic of China. Yonghai Chen, Chinese Academy of Sciences, P.0. Box 912, Beijing 100083, Peoples Republic of China; 86-10 8230-4227: fax 86-10-8230-5052; e-mail yhchen@red.semi.ac.cn simc.semi.ac.cn:6666.

21-24 Intl. Conf. on Semi Solid Processing of Alloys and Composites, S2P 2004, Limassol, Cyprus. Carol Garofoli, Worcester Polytechnic Institut, 100 Institute Road, Worcester, MA, 01609-2280; 508-831-5592; fax 508-831-5993. e-mail s2p@wpi.edu; www.wpi.edu/News/Conf/S2P.

22-24 $\nabla$ 3rd Annual Integrated Nanosystems: Design, Synthesis, and Applications Conf. \&

Nanotechnology Showcase,

Pasadena, CA. ASME Nanosystems, e-mail nano@asme.org;

www.asmeconferences.org/nano04.
27-29 $\nabla$ Photonics North 2004 Ottawa, ON SPIE, SPIE Meetings, P.0. Box 10, Bellingham, WA 98227-0010; 360-676-3290; fax 360-647-1445; e-mail spie@spie. org; spie.org/conferences/ calls/04/pn.

\section{OCTOBER 2004}

3-5 $\nabla$ 11th Intl. Workshop on Oxide Electronics (WOE11) Kanagawa, Japan. WOE11, woe11.msl.titech.ac.jp.

3-8 206th Meeting of the Electrochemical Society, Honolulu, HI. The Electrochemical Society, 65 S. Main St., Bldg. D, Pennington, NJ 08534-2839; 609-737-1902; fax 609-737-2743; e-mail ecs@ electrochem.org; www.electrochem. org/meetings/meetings.htm.

3-8 Applied Superconductivity Conf. (ASC/04), Jacksonville, FL. ASC 2004, www.ascinc.org

4-7 23rd Intl. Conf. on Applications of Lasers \& ElectroOptics (ICALEO 2004), San Francisco, CA. LIA Conf. Dept. e-mail conferences@laserinstitute. org; www.icaleo.org.

6-8 7th Intl. and Interdisciplinary Symp. of the Working Group on

"Biomaterials and Tissue Compatibility," Essen, Germany. Universität Duisburg-Essen, Alfons Fischer, Werkstofftechnik II, Lotharstr. 1, 47057 Duisburg, Germany; 49-203-379-4373; fax 49-203-379-4374; e-mail wtechii@uni-duisburg.de.

7-8 14th Intl. Activated Carbon Conf., Pittsburgh, PA. Henry Nowicki, e-mail hnpacs@aol.com; members.aol.com/hnpacs/ conferences/IACC14.htm

10-13 $\nabla$ 41st Annual Technical Meeting of the Society of Engineering Science: Symp. on Micro- and Nanoscale Laser Materials Processing, Lincoln, NE. Yongfeng Lu, e-mail ylu2@unl.edu; www.nuengr.unl.edu/ses2004/ symposia/mnmc.html.

10-14 Frontiers in Optics 2004 Rochester, NY. Robert Boyd, University of Rochester, Rochester NY, www.frontiersinoptics.org.

17-24 PM2004 Powder Metallurgy World Congress and Exhibition, Vienna, Austria. EPMA, Old Bank Buildings, Bellstone, Shrewsbury, United Kingdom 44-1743-248899; fax 44-0-1743362968; e-mail fh@epma.com; www epma com/pm_2004/ technical_program.htm.

18-21 $\nabla 2004$ IEEE Integrated Reliability Workshop (IRW 2004), S. Lake Tahoe, CA. IRW 2004, www.irps.org/irw.

18-21 Materials Solutions 2004 Conf. and Exhibition, Columbus, OH. ASM Customer Service, 9639 Kinsman Road, 
8-12 $\nabla$ NanoSikkim II: Friction and Biotribology, Sikkim, India. Indian Institute of Sciences, M Bobji, Bangalore 560 012, India, 91-80-2293-3233; fax 91-80-2360 0648; e-mail nanosikkim@mecheng. iisc.ernet.in; www.nanotribology. org/nanosikkimii/Sikkim2004.htm

8-12 $\nabla$ SPIE Fourth Intl. AsiaPacific Environmental Remote Sensing Symp., Honolulu, HI. SPIE, SPIE Meetings, P.O. Box 10, Bellingham, WA, 98227-0010 360-676-3290; fax 360-647-1445 e-mail spie@spie.org; spie.org/conferences/calls/04/ae.

14-16 37th IMAPS Annua Symposium on Microelectronics, Long Beach, CA. IMAPS, 611 2nd St., N.E., Washington, DC 20002; 202-548-4001; fax 202-548-6115; www.imaps.org

14-19 $\nabla$ AVS 51st Intl. Symp. and Exhibition, Anaheim, CA. University of Washington, David Castner, Seattle, WA, 98195; email dave castner@avs.org; www.avs.org.

15-18 36th Intl. SAMPE Technical Conf., San Diego, CA. DeWayne Howell, CompositeTek Boulder, C0, 303-530-2919; fax 303-530-9437; e-mail dhowell@ compositetek.com; www.sampe.org

15-19 ASNT Fall Conf. \& Quality Testing Show 2004, Las Vegas, NV. Kelly Wise, 614-274-6003 fax 614-274-6899

e-mail kwise@asnt.org

www.asnt.org/events/conferences fc04/prospectus.pdf.

16-18 Intl. Conf. in Asia (IUMRS-ICA 2004), Hsinchu, Taiwan. Grace Lee, Materials Research Laboratories, ITRI, e-mail ica2004@itri.org.tw; http://www.ica2004.org.tw. IUMRS/MRS-T.

24-26 1st Intl. Symp. on Shape Memory and Related Technologies (SMART 2004), Singapore. Yong Liu, Nanyang Technological Univ. Nanyang Ave., Singapore; 65-6790 4951; e-mail mliuy@ntu.edu.sg; www.ntu.edu.sg/home/mliuy/ SMART2004.htm.

24-26 3rd Intl. Conf. on Materials Processing for Properties and Performance (MP3)/Intl. Conf on Nanostructures and Nanotechnology (N2), Singapore. K. Khor, Nanyang Technological Univ., 50 Nanyang Ave., Singapore 65-6-791-1859; fax 65-6-790-5526; e-mail mkakhor@ntu.edu.sg; www.materials.org.sg.

29-3 MRS Fall Meeting, Boston, MA. Materials Research Society, 506 Keystone Dr., Warrendale, PA 15086-7573; 724-779-3003; fax 724-779-8313; e-mail info@mrs. org; www.mrs.org. MRS.

\section{DECEMBER 2004}

2-2 $\nabla$ SPIE Rapid Design Engineering, and Moldmaking Conf: Reduce Time to Market, Frankfurt, Germany. Society of Plastics Engineers, Yetty Pauwels, Bistkapellei 44, Antwerp, Belgium, 32-3-541-77-55: fax 32-3-541-8425; e-mail spe.europe@pi.be; www.4spe.org/semcont/

conferences/conferences.htm.

6-8 Intl. Symp. on Advanced Materials and Processing,

Kharagpur, India. A.K. Banthia, Indian Institute of Technology, Kharagpur, India, 91-3222-282274; e-mail akb@matsc.iitkgp.ernet.in www.iitkgp.ernet.in/departments/ home.php?deptcode=MS.

\section{JANUARY 2005}

10-14 IUVSTA Workshop on One-Dimensional Nanomaterials, Taipei, Taiwan. R. Chu-Chen, 886-2-33665202: fax 886-223655404; e-mail iuvsta@ms. ntu-ccms.ntu.edu.tw.

22-27 $\boldsymbol{\nabla}$ Photonics West 2005 San Jose, CA. SPIE, SPIE Meetings, P.0. Box 10, Bellingham, WA 98227-0010; 360-676-3290; fax 360-647-1445; e-mail spie@spie.org; spie.org/Conferences/calls/05/pw.

\section{FEBRUARY 2005}

6-11 Second Intl. Conf. on Advanced Materials and Nanotechnology (AMN-2), Conference Secretariat, University of Canterbury, Private Bag 4800, Christchurch, New Zealand: 64-3-364-2534; fax 64-4-364-2324; e-mail amn2@cont.canterbury.ac. nz; www.macdiarmid.ac.nz/amn-2.

24-26 Small World Expo, Las Vegas, NV. Jim Sugerman, 800-342-2032; www.

\section{MARCH 2005}

6-9 Materials for Advanced Metallization 2005 (MAM2005), Dresden, Germany. MAM2005, 49-371-531-3130; e-mail info@ mam-conference.org; www. mam-conference.org.

14-18 ASNT Spring Conf. and 14th Annual Research Symp., Albuquerque, NM. Kelly Wise, 614-274-6003; fax 614-274-6899; e-mail kwise@asnt.org.

21-25 2005 American Physical Society March Meeting, LoS Angeles, CA. APS. Queenstown, New Zealand. smallworldexpo.com
28-1 MRS Spring Meeting, San Francisco, CA. Materials Research Society, 506 Keystone Dr. Warrendale, PA 15086-7573; 724779-3003; fax 724-779-8313; e-mail info@mrs. org; www.mrs.org. MRS.

\section{APRIL 2005}

4-5 $\boldsymbol{\nabla}$ OPT0-Ireland 2005 Dublin, Ireland. SPIE, SPIE Meetings, P.0. Box 10, Bellingham, WA, 98227-0010; 360-676-3290; fax 360-647-1445; e-mail spie@spie.org spie.org/conferences/calls/05/ire.

4-8 2005 Intl. Magnetics Conf. (INTERMAG2005), Nagoya, Japan. ntermag 2005 Secretariat, c/0

Convention Linkage, Inc., AkasakaNihon Bldg., 9-5-24, Akasaka, Minato-ku, Tokyo 107-0052, Japan 81-3-5770-5531; fax 81-3-57705532; e-mail info@intermag2005.jp; www.intermag2005.jp. Endorsed.

11-14 Fourteenth Intl. Conf. on Microscopy of Semiconducting Materials, Oxford, United Kingdom. Univ. of Sheffield, Tony Cullis, Sheffield, United Kingdom, 44-114-222-5407; fax 44-114272-6391; e-mail a.g.cullis@ sheffield.ac.uk; www.rms.org.uk Endorsed.

24-28 $\nabla$ 15th Intl. Conf. on Wear of Materials, San Diego, CA. Gill Heaton, Secretariat, Hillside Cottages, Wheatley Road, Islip, Oxford OX5 2TF, UK; 44-0-1865373625; fax 44-0-1865-375855; e-mail wom-conference@elsevier. com; www.wom-conference. elsevier.com.

\section{MAY 2005}

29-3 $\nabla$ 2nd Intl. Conf. on Electrophoretic Deposition: Fundamentals and Applications, Barga, Italy. Impe. C. of Sci, Tech. \& Med., A Boccaccini, London, United Kingdom, 44-207-5946731; fax 44-207-5843194;

e-mail a.boccaccini@imperial.ac.uk; www.engconfintl.org/5ad.html.

\section{JUNE 2005}

26-29 17th University Conf. on Glass Science, University Park, PA. Carlo Pantano.

\section{JULY 2005}

3-8 Intl. Conf. on Materials for Advanced Technologies (ICMAT 2005) and IUMRS-ICAM 2005, Singapore Mat Research Soc (Singapore), ICMAT Secretariat, 3 Research Link, Singapore, Singapore, 65-6874-1975; ax65-6777-2393; e-mail icmat@ mrs.org.sg; www.mrs.org.sg/ meeting_future.html. IUMRS/MRS-S
4-8 Lucerne Fuel Cell Forum 2005, Lucerne, Switzerland. EFCF, Morgenacherstrasse 2F, P.0. Box 99 Oberrohrdorf, Switzerland, 41-56496-7292; fax 41-56-496-441; e-mail forum@efcf.com.

10-15 $\nabla$ 16th Intl. Conf. on Electronic Properties of Two Dimensional Systems (EP2DS-16), Albuquerque, NM. Jerry Simmons, 505-844-8402; e-mail jsimmon@ sandia.gov. Endorsed.

17-22 Intl. Conf. on Solid State Ionics (SSI-15), Baden-Baden, Germany. Ellen Ivers-Tiffee, c/0 Universität Karlsruhe, Kaiserstr 12, D-76131 Karlsruhe, Germany; 49-721-608-7491; fax 49-721-6087492; e-mail info@ssi-15.net; www.ssi-15.net.

19-23 5th Intl. Conf. on Intelligent Processing and Manufacturing of Materials, Monterey, CA. IPMM 05 , www.mining.ubc.ca/ipmm.

\section{AUGUST 2005}

28-2 6th Intl. Conf. on Nitride Semiconductors (ICNS-6), Bremen, Germany. Sven Einfeldt, University of Bremen, P. O. Box 330440 Bremen, Germany; 49-421-218 7453; fax 49-421-218-4581; e-mail icns6@ifp.uni-bremen.de; www.ifp.uni-bremen.de/icns6.

\section{OCTOBER 2005}

2-6 $\nabla$ 3rd Fracture and Flow in Advanced Glasses Conf.

University Park, PA. Carlo Pantano. Quality Testing Show 2005 Columbus, $\mathrm{OH}$. Kelly Wise, 614274-6003; fax 614-274-6899; e-mail kwise@asnt.org.

\section{NOVEMBER 2005}

22-25 $\nabla$ Ninth NCB Intl. Seminar on Cement, New Delhi, India. NCCBM, Ninth NCB, 34 Km Stone, Delhi Mathura Road (NH2), Ballabgarh 121 004, Haryana, India, 91-129-2242051-56; fax 2242100, 2246175; e-mail nccbm@giasd101.vsnl.net.in.

\section{9-3 $\nabla$ MRS Fall Meeting,} Boston, MA. Materials Research Society, 506 Keystone Drive, Warrendale, PA 15086-7573; 724-779-3003; fax 724-779-8313; e-mail info@mrs.org; www.mrs.org. MRS.

\section{DECEMBER 2005}

5-7 $\nabla$ 2nd Intl. Conf on Advances in Production and Processing of Aluminum (APPA 2005), Bahrain. Conf. Secretariat (APPA 2005), Univ. of Bahrain, P.0. Box 32038, Bahrain, 973e-mail appa2005@eng.uob.bh; www.uob.edu.bh/colleges/ engineering/conf/index.htm.
17-21 ASNT Fall Conf. and 17876114; fax 973-17876655;

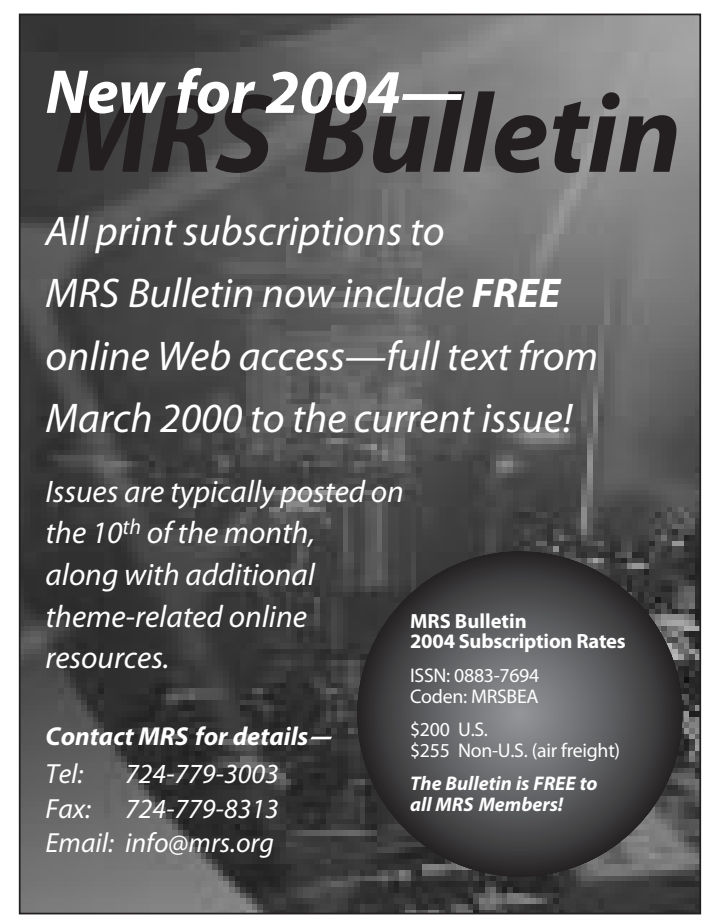

